\title{
Design of Adaptive RFID Reader based on DDS and RC522
}

\author{
Li Yang, Dong Zhi-Hong, Cong Dong-Sheng
}

Beijing Key Laboratory of Signal and Information Processing for High-end Printing Equipments,

Beijing Institute of Graphic Communication

\#1 Xinghua Avenue, Daxing District, Beijing, China 102600

yangli@bigc.edu.cn

Keywords: RFID, Adaptive Frequency, DDS, AD9854

\begin{abstract}
In traditional Radio Frequency Identification (RFID) reader system, the electromagnetic energy cannot be transferred as much as possible, which is caused by the discrete resonance frequency of antenna. The energy transferred distance should be reduced by this reason. In this paper, Direct Digital Synthesizer (DDS) technique and the adaptive frequency work mode are applied in RFID reader. Under this condition, the resonance frequency of RFID tag can be searched automatically by the reader. Therefore, the resonance frequency error can be decreased, and the match between reader and tag can also be optimized. As the result, the reading distance of RFID system is expanded. In this paper, the RFID system is controlled by STC8952, and DDS is realized by AD9854.
\end{abstract}

\section{Introduction}

RFID system has been applied in the world wide, and RFID is also one research focus. DDS is a type of frequency synthesizer used for creating arbitrary waveforms from a single, fixed frequency reference clock. DDS has many advantages over its analog counterpart, the phase-locked loop, including much better frequency agility, improved phase noise, and precise control of the output phase across frequency switching transition ${ }^{[1]}$.

In this paper, DDS technique is applied to control RC552 change frequency. Therefore, the RFID reader can track the resonance frequency of RFID tag automatically, and then, both coupling efficiency and identification distance are improved. The anti-collision algorithm is also introduced to stabilize the reading process.

\section{Method of frequency matching}

According to the international standards for close-coupled IC card (such as ISO/IEC 14443-TYPE $\mathrm{A}$, and ISO/IEC 14443-TYPE B), the resonance frequency of reader and tags should be perfect matched. In this case, the identification sensitivity of RFID system could be max as possible. However, in experience, these two resonance frequencies cannot be matched as wanted cause the error of tag antenna and the surrounding interference. The reading distance of RFID system can be reduced by the mismatching ${ }^{[2]}$.

To resolve the problem of mismatching, some manufactories applied the addition trimmer capacitor in the tag chip to offset the tag antenna error. Nevertheless, the influence from environment interference cannot be decreased in this way ${ }^{[3]}$.

To improve the frequency matching between reader and tag, the output frequency of RFID reader is designed to be flexible and swept in certain range in this paper. While the alternating frequency $f_{\mathrm{c}}$ from reader is equal to the resonance frequency $f_{\mathrm{r}}$ from tag, the oscillation circuit at tag is resonated. The resonant process can be observed by the short-term voltage or current change in resonant coil. The current increase or voltage decrease is called as Dip. The relative intensity of Dip is responded to the distance between coils and their relative moving speed ${ }^{[4]}$. An obviously Dip is need to guarantee the reliable identification.

The sweeping frequency can help the reader efficiently find the matched frequency point while 
tag closing. The output frequency of the reader is swept from the minimum frequency point to the maximum frequency point. While the output frequency is exactly equal to the tag resonance frequency, the current in the tag coil can produce an obviously Dip. In this way, the communication between reader and tag is achieved.

According to the relative standards, the center frequency error of RFID tag should be limited in $5 \%{ }^{[5]}$. As the center frequency of $13.56 \mathrm{MHz}$, the sweeping frequency range is from $12.882 \mathrm{MHz}$ to $14.238 \mathrm{MHz}$. The data transfer velocity is $106 \mathrm{k} \mathrm{bit} / \mathrm{s}$, and the data bit week is $9.4 \mathrm{ps}$. In the general scanning procedure, the faster sweeping speed leads the shorter identify distance, and the slower sweeping speed leads the longer identify period. To balance the identify distance and period, in this paper, the frequency sweeping week is set to be $5 \mathrm{~ms}$ which include 500 data weeks. The amplitude of DDS output signal is between 200 500mVpp, which cannot drive the following digital chips and need to be amplified to satisfy the requirement of TTL driving circuit.

\section{Hardware Design}

The system (shown in Fig.1) is controlled by MCU of STM32, which drive AD9854 for frequency sweeping, and also provide the clock for RC522 read-write module. LCD and memory chip are also mounted for state display and data restore in this system.

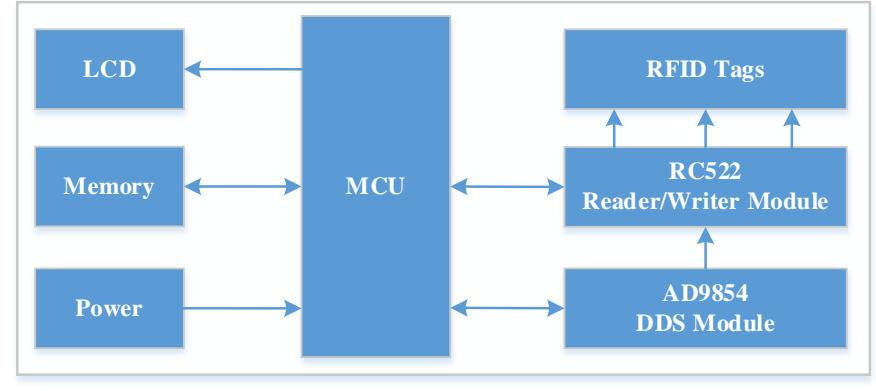

Fig.1 System Architecture

Design of the Rx-Tx module based on RC522. The modulation circuit is exist in RC522, and the external carrier is do not necessary. Considering the carrier wave is provided by crystal oscillator in RC522, the carrier frequency is equal to the clock frequency. In this case, the sweeping signal can be sent to OSC IN pin of RC522 directly, and replace the formal stable crystal, which solve the problem of carrier wave changing for ASK modulation. However, this also introduces the jitter on internal clock and its phase. Considering the asynchronization communication of RF system, the tiny change on clock signal cannot influence on data extraction and transmission.

The Rx-Tx module based on RC522 is shown in Fig.2. RC522 is connected with MCU by 8-pin transmission line, which include SDA/SCLK (as serial control bus), and MOSI/MISO (as communication interface $)^{[6]}$. In this design, the EMC low pass filter is realized by multi-layer PCB board. During receiving period, the respond signal from tag can be modulated from double-side band of subcarrier. To stabilize the output of VMID, which used to be the bias of Rx input, the capacitor of C22 need to connect VMID and GND. The receiver circuit need one voltage divider between Rx and VMID, and this is realized by R4, RS and C25. 


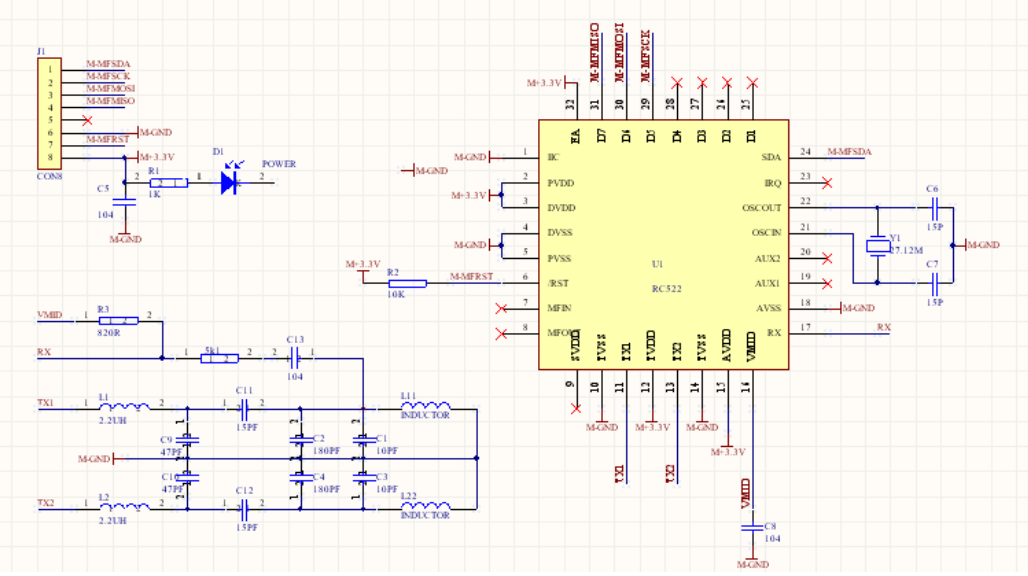

Fig.2 Rx/Tx Module based on RC522

Design of DDS frequency sweeper based on AD9854.The DDS technique is based on Nyquist theory. According to the phase of continual signal, one sinusoidal signal can be sampled, quantized and encoded into a sinusoidal function table, and restored in EPROM. While synthesizing, the phase variation can be changed by the frequency control bit in the phase accumulator. The different phase variation leads different sampling time during one week ${ }^{[7]}$. In this method, the output frequency can be changed while clock frequency and sampling frequency are not changed.

AD9854 is controlled by two part I/O interfaces, which is shown in Fig.3. One part is used to control data flow, the other one is used to control address and special functions. In this design, the output is separated into two parts, and both connected with low pass filter, which guarantee signal smoothly transmitting.

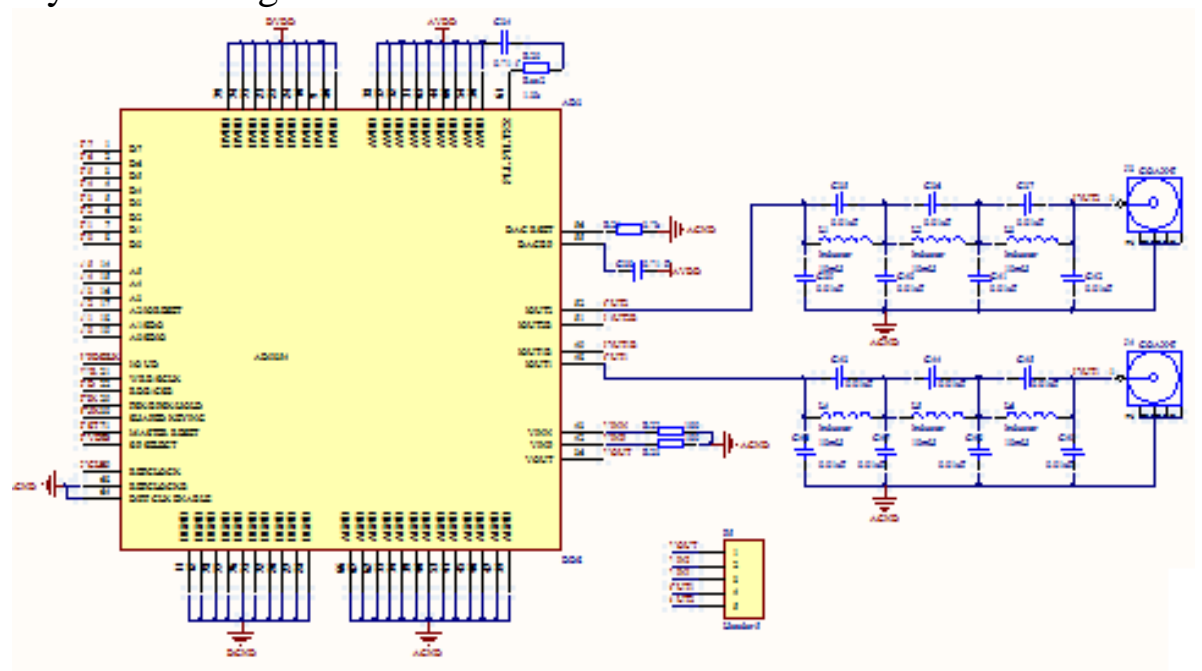

Fig.3 Frequency Sweeper based on AD9854

MCU Control System.In this design, STC8952 is used as MCU, which can control AD9854 as frequency sweeper, and control RC522 to read/write tag. The information on tag can be displayed on LCD.

Considering the complex control on AD9854, the interfaces of P0 and P2 are used to control frequency sweeper, and P1 is used to control RC522 and LCD. P3 is used to control keyboard, memory and serial port. 


\section{Algorithm Design}

The whole system (shown in Fig.4) consists of main program, RC522 driver and LCD driver. The main program defines the I/O interfaces, head files and character array. At the beginning of main program, LCD and AD9854 are initialed. And DDS send out sweeping frequency from $12.882 \mathrm{MHz}$ to $14.238 \mathrm{MHz}$ by register setting, until the interrupt coming. At the same time, RF read/write module operate at the mode of tag seeking. While one tag is detected, and the maximum frequency response is reached, the output frequency of sweeper is maintained at current frequency. Then, the read/write operation is proceed, and the related information is displayed on LCD. All of above is one entire tag read/write procession.

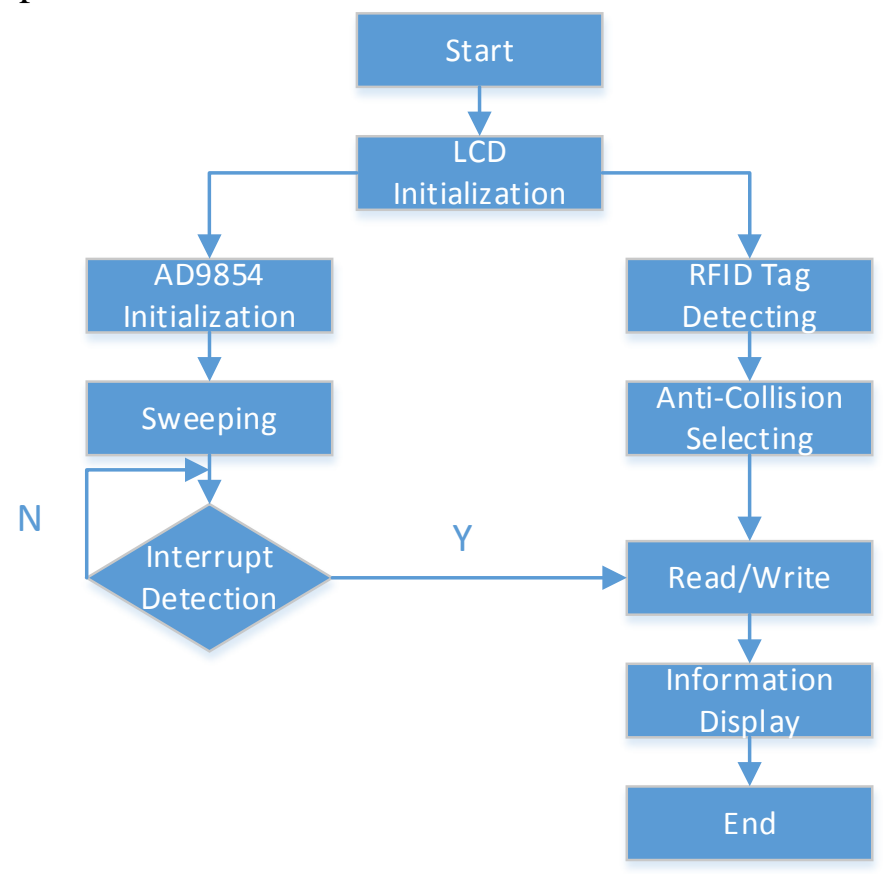

Fig.4 Program Flow Diagram for System

AD9854 Control Procedure.Similar with the main program, the first step for AD9854 control program is initialization. The following step is to send the control word to AD9854 at the rising edge of clock signal, and the write function is applied. The control word is stored in register. The next step is read data from AD9854 at the falling edge of clock signal, and the read function is used.

RC522 Control Procedure. While the tag is detected by antenna, RC522 send the request command. If the handshake signal is responded from the tag, the communication between the reader/writer and tag is confirmed. To prevent the data collision caused by large quantities of tags detected, the anti-collision algorithm is applied. In this algorithm, each tag own the unique serial number, which can be cyclic checked by MCU and produce one verification number. The verification number is send back to tag, and while this number is confirmed to be right by tag, the selection of tag is confirmed. After this selection procedure, the read/write operation can be proceed.

\section{System Test}

While the interrupt signal from RFID driver is collected by MCU, the sweeper is stopped, and the output frequency is also stopped at the optimized resonance frequency point until the read/write operation completed. In this design, the identification distance is improved than traditional RFID system. In experience, the identification distance of new system (shown in Fig.5) based on DDS sweeper can reach to $10 \mathrm{~cm}$. This is much better than traditional system based on fixed frequency, which can detect tag at the distance of $7 \mathrm{~cm}$ in the same environment. 


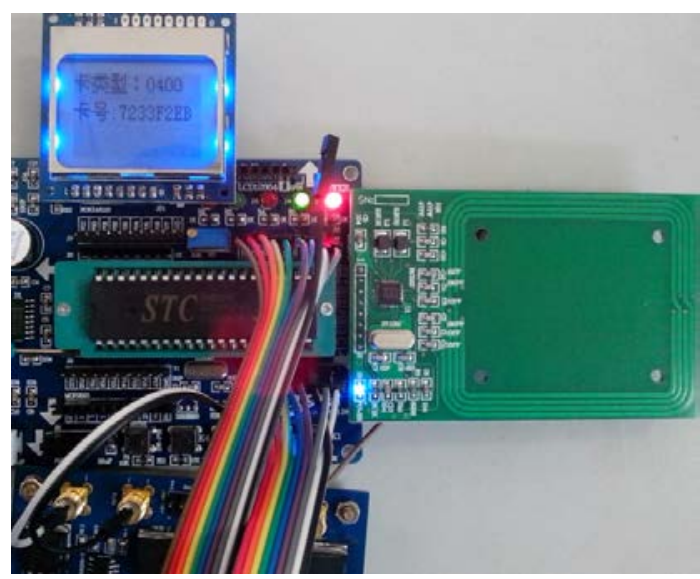

Fig.5 RFID Read/Write System

\section{Conclusion}

In this paper, the adaptive frequency RFID reader/writer is designed and manufactured. Based on the test result, it's proven that the frequency sweeper based on DDS can improve the detection distance of RFID reader/writer. DDS frequency sweeper reduces the influence from the discrete frequency of resonance circuit on antenna built in RFID tag, and the frequency offset caused by working environments.

\section{Acknowledgements}

This work was financially supported by the BIGC General Science Program (23190113082), BIGC Talent Program (27170115004/038).

\section{References}

[1]. Single-chip direct digital synthesis vs. the analog PLL. Analog Devices Corporation.

[2]. Klaus Finkenzeller. RFID Handbook Fundamentals and Applications in Contactless Smart Cards and Identification. Second Edition. John Wiley \& Sons Ltd, 2003:83

[3]. Antenna Circuit Design for RFID Applications. Microchip Technology Inc. 2003:12-14

[4]. Proximity Antenna Training for $13.56 \mathrm{MHz}$ Directly matched antenna. Philips Semiconductors. $20031-3$

[5]. ISO 14443 Identification cards - Contactless integrated circuits cards - Proximity cards, International Organization for Standardization Publication Date, Feb l, 2001: Part 1-4

[6]. Data Sheet MIFARE MF RC500 Highly Integrated ISO 14443A Reader IC, Revision 2.0, Philips semiconductors, January 2002

[7]. L.J.Kushner. The Composite DDS - a new direct digital synthesizer architecture. IEEE 47th International Frequency Control Symposium. 1993 